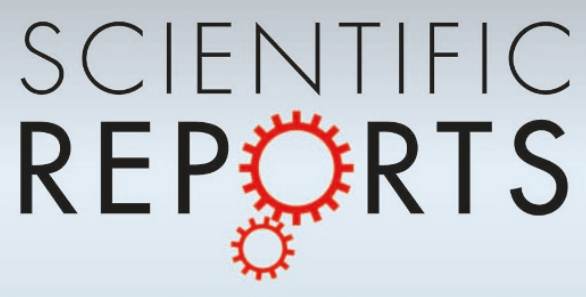

OPEN

SUBJECT AREAS:

TERAHERTZ OPTICS

ULTRAFAST PHOTONICS

NANOPHOTONICS AND

PLASMONICS

SUB-WAVELENGTH OPTICS

Received

13 December 2012

Accepted

14 February 2013

Published

6 March 2013

Correspondence and requests for materials should be addressed to J.M. (juliette. mangeney@u-psud.fr)

\section{Subwavelength metallic waveguides as a tool for extreme confinement of $\mathrm{THz}$ surface waves}

\author{
D. Gacemi, J. Mangeney, R. Colombelli \& A. Degiron
}

Institut d'Electronique Fondamentale, Univ. Paris Sud, UMR CNRS 8622, 91405 Orsay, France.

Research on surface waves supported by metals at $\mathrm{THz}$ frequencies is experiencing a tremendous growth due to their potential for imaging, biological sensing and high-speed electronic circuits. Harnessing their properties is, however, challenging because these waves are typically poorly confined and weakly bound to the metal surface. Many design strategies have been introduced to overcome these limitations and achieve increased modal confinement, including patterned surfaces, coated waveguides and a variety of sub-wavelength geometries. Here we provide evidence, using a combination of numerical simulations and time-resolved experiments, that shrinking the transverse size of a generic metallic structure always leads to solutions with extreme field confinement. The existence of such a general behavior offers a new perspective on energy confinement and should benefit future developments in $\mathrm{THz}$ science and technology.

$\mathrm{T}$ he manipulation of THz surface modes, known as Sommerfeld waves for cylindrical/wire waveguides ${ }^{1,2}$ and Zenneck waves for planar surfaces ${ }^{3}$ has been a long-standing challenge due to the two conflicting roles played by metals in these systems. On one hand, these modes exist because metals have a good conductivity: they result from the coupling between electromagnetic waves and moving charges at the surface of the conductor ${ }^{4}$. On the other hand, this interaction is typically weak because the same charges have a screening effect that severely limits the penetration of the electromagnetic fields inside the metal. Thus, the modes are poorly bound; for smooth metallic waveguides, electric fields associated with $\mathrm{THz}$ surface waves typically penetrate $\sim 50-100 \mathrm{~nm}$ into the metal but extend tens of wavelengths (i.e. hundreds of microns) into the dielectric above. In fact, this problem is not specific to the $\mathrm{THz}$ regime and it is even more acute at gigahertz and radio frequencies.

In 1950 Goubau predicted that the adverse effects of the metal conductivity on $\mathrm{THz}$ and other low frequency waves could be mitigated by either structuring the surface along which the modes propagate or by coating the metal with a dielectric layer of finite thickness ${ }^{5}$. He demonstrated that in both cases, the modified surface could be seen as having a much lower effective conductivity than the metal alone, leading to well-confined solutions even in the limit of a perfectly conducting core. Since this pioneering work, several studies have confirmed that the modes supported by metal waveguides with subwavelength corrugations, known as "spoof plasmons" in the modern literature, are more tightly bound than conventional Zenneck or Sommerfeld waves ${ }^{6-11}$. Likewise, many authors have demonstrated that improved field confinement could be obtained by coating metal surfaces with a thin dielectric film ${ }^{4,12-15}$. In recent years, the community realized that other strategies could be used to create highly sub-wavelength modes, for example by working with micrometer-size metal tip apexes ${ }^{16,17}$, metallic nanoslits ${ }^{18,19}$, small diameter single wires ${ }^{20,21}$, parallel-plate waveguides with micrometer-size width and separation ${ }^{22-24}$, and planar Goubau lines ${ }^{25-27}$.

Thus, it appears that a variety of phenomena and approaches are readily at hand to confine THz surface waves into highly sub-wavelength volumes. In this article we show that the existence of strongly confined modes in all these aforementioned geometries has in fact the same origin, independent from the physical mechanisms that bind the waves to the metal surface: the reduction of the waveguide size in at least one direction transverse to the propagation. To see this phenomenon at work, we model and fabricate structures that support THz surface modes through very different physical principles: a single Au stripe embedded in a homogeneous medium, a perfectly conducting stripe supported by a dielectric layer with a finite thickness, and a planar Goubau line.

\section{Results}

Modes supported by a Au stripe in a symmetric environment. We examine the guiding properties of a rectangular Au stripe embedded in a homogeneous matrix with a dielectric index $n=1.46$ (Fig. 1a). This 
a
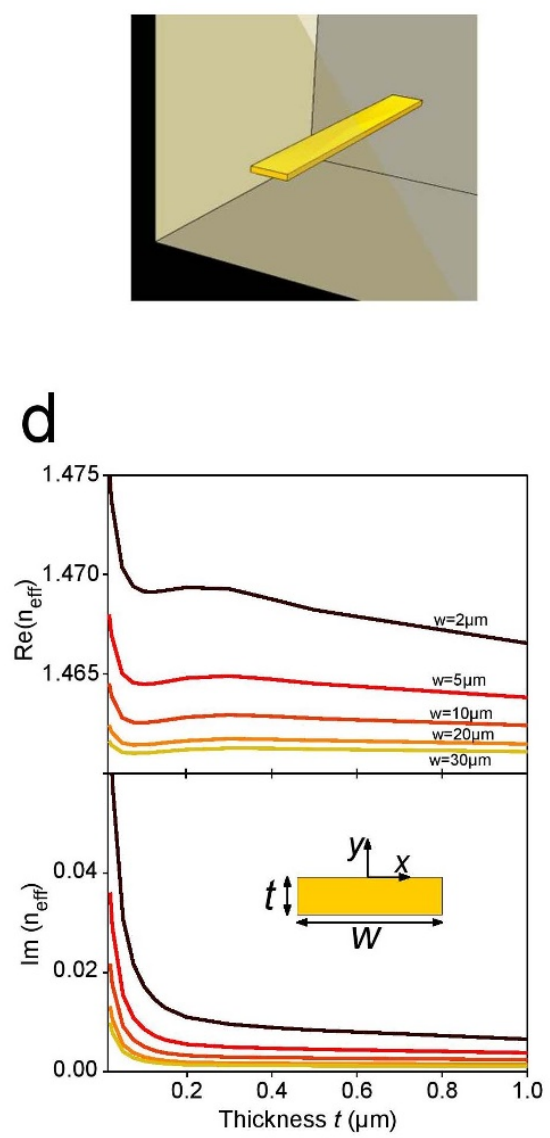

b
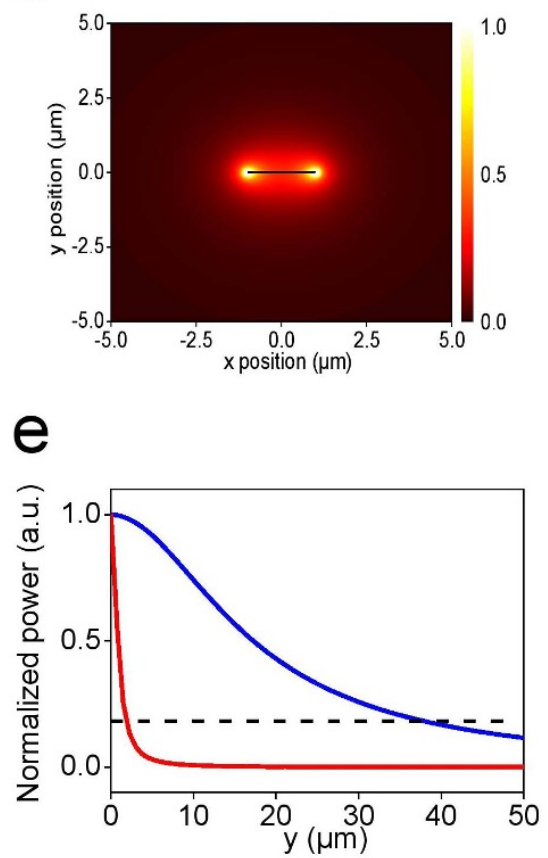

C

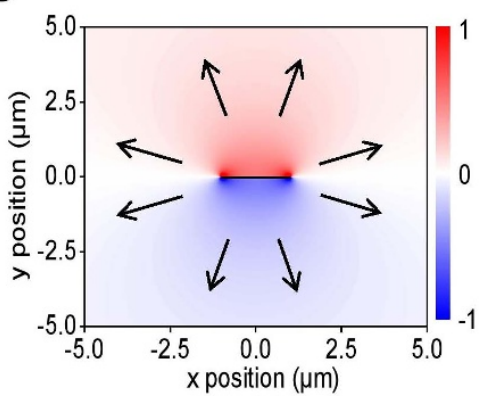

f

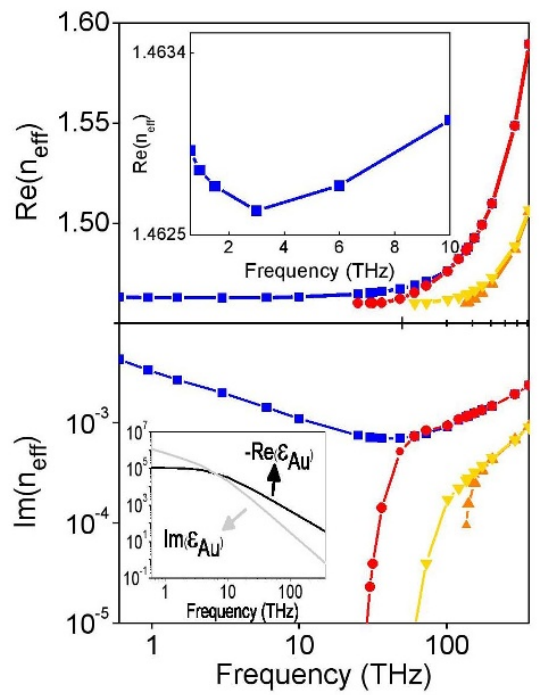

Figure 1 Simulation of the modes supported by a Au stripe in a symmetric environment. (a) Schematic of the Au waveguide embedded in a homogeneous dielectric environment. (b) Cross-section map representing the power carried by the mode propagating along a Au stripe with a width $\mathrm{w}=2 \mu \mathrm{m}$ and a thickness $\mathrm{t}=20 \mathrm{~nm}$ at $1 \mathrm{THz}$. The calculations have been performed at $1 \mathrm{THz}$ but no other mode has been found by sweeping the frequency from $0.5 \mathrm{THz}$ to $20 \mathrm{THz}$ or by increasing the lateral dimensions of the stripe up to a few hundreds of microns. (c) Ey field distribution for the same mode. The arrows represent the total field E. (d) Real (top) and imaginary parts (bottom) of the effective index neff as a function of the stripe thickness t. The different curves correspond to stripes with different widths $\mathrm{w}$, as indicated on the graph. The smallest thickness used in the simulations is $\mathrm{t}=0.02 \mu \mathrm{m}$. Although it is possible to simulate thinner stripes as well, we note that real Au films become extremely rough for $\mathrm{t}<0.02 \mu \mathrm{m}^{34}$.

(e) Cross-section of the power carried by the mode for two waveguides of different sizes. Blue curve: large stripe with a width $\mathrm{w}=30 \mu \mathrm{m}$ and a thickness $\mathrm{t}=10 \mu \mathrm{m}$; Red curve: small stripe with $\mathrm{w}=2 \mu \mathrm{m}$ and $\mathrm{t}=20 \mathrm{~nm}$. Here the power has been evaluated along the vertical symmetry plane of the structure (i.e. the y axis). (f) Dispersion relation of the modes supported by a Au stripe with a width $\mathrm{w}=10 \mu \mathrm{m}$ and a thickness $\mathrm{t}=200 \mathrm{~nm}$, corresponding to typical dimensions of $\mathrm{THz}$ metallic structures reported in literature as Goubau lines. Only the first lowest order modes have been represented and labeled according to the nomenclature of Ref.28. Blue curve: short-range $\mathrm{sa}_{\mathrm{b}}{ }^{0}$ mode, red curve: short-range $\mathrm{aa}_{\mathrm{b}}{ }^{0}$ mode, orange curve: long-range as ${ }_{\mathrm{b}}{ }^{0}$ mode and yellow curve: long-range $\mathrm{ss}_{\mathrm{b}}{ }^{0}$ mode. The top inset represents a close-up of $\operatorname{Re}\left(\mathrm{n}_{\mathrm{eff}}\right)$ for the $\mathrm{sa}_{\mathrm{b}}{ }^{0}$ mode in the THz range while the bottom inset shows the permittivity of Au across the electromagnetic spectrum (data taken from Ref. 33). Note that this permittivity provides an accurate description of Au from the $\mathrm{THz}$ to the mid-infrared range but that it slightly underestimates the losses at higher frequencies compared to other experimental datasets ${ }^{35,36}$.

geometry admits solutions that result from the sole coupling between electromagnetic fields and the free electrons at the metal surface and we investigate its properties with a finite element package (cf Supplementary Methods).

We first consider a Au stripe with a width $w=2 \mu \mathrm{m}$ and a thickness $t=20 \mathrm{~nm}$. Figs. $1 \mathrm{~b}$ and $1 \mathrm{c}$ show the power distribution, the $\mathrm{E}_{\mathrm{y}}$ field pattern, and a plot of the vectors representing the electric field of the only mode found by our solver at $1 \mathrm{THz}$. In the remaining of this work, we use $\mathrm{E}_{\mathrm{y}}$ to identify the field symmetries of the solutions and note that the symmetries of the other components can be deduced from this information through Maxwell's equations. The mode of Figs. 1b-c is characterized by a strong concentration of the electromagnetic energy around the corners, which act as lightning rods; in addition, its field component $\mathrm{E}_{\mathrm{y}}$ is antisymmetric with respect to the $x$ axis and symmetric with respect to the $y$ axis. In fact, this mode can be seen as a sub-wavelength variant of a Sommerfeld wave: both solutions are bound to a single metallic line in a homogeneous medium and both are characterized by an axial field distribution.

To get a deeper insight, we examined how the mode properties depend on the dimensions and aspect ratio of the rectangular waveguide. Fig. 1d summarizes the results by plotting the dispersion of the mode as a function of the metal thickness $t$ at $1 \mathrm{THz}$. Several curves are shown corresponding to waveguides of different widths $w$. In all cases, the real part of the effective index $n_{\text {eff }}$ follows three successive trends as $t$ decreases: a continuous rise until $t$ reaches approximately $0.3 \mu \mathrm{m}$, a slight sag, and finally a sharp upturn for thicknesses smaller than approximately $0.1 \mu \mathrm{m}$. In this latter regime, the slope of the curves becomes markedly steeper. This behavior is all the more marked than the width of the waveguide is also small. Further calculations, not shown here, indicate that the intermediate regime $(0.3 \mu \mathrm{m}>\mathrm{t}>0.1 \mu \mathrm{m})$ is caused by the conductivity of the Au stripe 
that temporary counters the dispersive effects induced by $t$. However, the geometrical parameters always end up overcoming the influence of the conductivity since all the curves of Fig. 1d diverge for metal thicknesses smaller than $0.1 \mu \mathrm{m}$-a value close to the skin depth of Au at $1 \mathrm{THz}(\sim 73 \mathrm{~nm})$. The imaginary part of $\mathrm{n}_{\text {eff }}$, has a smoother evolution but also diverges for vanishing Au thicknesses. These dispersion curves consistently demonstrate that the mode evolves into a highly confined solution as the transverse dimensions of the metal waveguide tend to zero. As always with metallic structures, this field confinement leads to increased absorption losses.

Fig. 1e shows a cross-section of the power carried by the mode along the $y$ axis for a relatively large metal stripe (width $w=30 \mu \mathrm{m}$, thickness $t=10 \mu \mathrm{m}$ ) and one with highly sub-wavelength dimensions $(w=2 \mu \mathrm{m}, t=20 \mathrm{~nm})$. One can see that the confinement dramatically increases as the waveguide cross-section is reduced. In particular, the distance at which the power decreases to $1 / 2 \mathrm{e}$ of its initial value reaches $\lambda / 93$ for the smallest stripe, where $\lambda=\lambda_{0} / 1.46 \sim$ $200 \mu \mathrm{m}$ is the wavelength of light in the dielectric medium, further illustrating that reducing the waveguide transverse size down to subwavelength scales leads to extreme field confinement.

Before elaborating further on this behavior, it is useful to note that a short-range surface plasmon characterized by the same field symmetries and by a similar increase of $\mathrm{n}_{\text {eff }}$ with shrinking lateral dimensions has been predicted by Berini in the near-infrared and visible part of the spectrum ${ }^{28,29}$. In the optical regime, the influence of the waveguide dimensions on $\operatorname{Re}\left(\mathrm{n}_{\mathrm{eff}}\right)$ and $\operatorname{Im}\left(\mathrm{n}_{\mathrm{eff}}\right)$ is explained by considering that the modes result from the coupling between the fields bound to the four walls of the metal waveguide. In addition, the presence of sharp corners further increases the confinement through lightning rod effects.

The $\mathrm{THz}$ surface mode naturally evolves towards the optical short-range plasmon of Ref.28, as can be verified by plotting their dispersion relation (Fig. 1f). We can therefore categorize our $\mathrm{THz}$ surface wave using Berini's nomenclature, i.e. we identify this solution as the $\mathrm{sa}_{\mathrm{b}}{ }^{0}$ mode. Importantly, it should be noted that many others surface plasmon modes exist at optical frequencies ${ }^{28}$. The dispersion relation of some of these solutions is also represented on Fig. 1f. One can see that as the frequency decreases, the real and imaginary parts of their refractive index decrease before experiencing a cutoff in the infrared. The $\mathrm{sa}_{\mathrm{b}}{ }^{0}$ mode is the only solution that survives in the $\mathrm{THz}$ range. Its effective index eventually raises again as the frequency reaches the $\mathrm{THz}$ regime (this increase can be better seen in the insert of Fig. If for the real part of the permittivity). Such unusual dispersion has already been observed some time ago for Sommerfeld waves propagating along cylindrical metal waveguides and has been attributed to conductivity effects ${ }^{30}$. This point can be further clarified by also taking into account the size effects. For a given geometry, such as the stripe of Fig. 1f, reducing the operation frequency has two consequences. First, it increases the real and imaginary part of the permittivity, resulting in a better conductivity that pushes the electromagnetic fields away from the metal surface. Second, the stripe becomes comparatively smaller with respect to the operation wavelength, and we know that a smaller cross-section reinforces the field confinement around the structure. Thus, the conductivity and the size effects have two competing roles. For the most part of the dispersion relation, from the visible to the far-infrared, the conductivity has a greater weight than the size effects because it increases by several orders of magnitude (inset of Fig. 1f). Consequently the modes become less confined which leads to a decrease of the real and imaginary part of the permittivity. In the $\mathrm{THz}$ range, however, the variations of the conductivity level off and the mode properties become dominated by the size effects. Consequently, reducing the transverse size of the waveguides down to subwavelength dimensions is a powerful tool to achieve high modal confinement. This conclusion can be extended to other waveguide geometries such as cylindrical wires.
Modes supported by a perfectly conducting stripe on a thin dielectric layer. We now show that the effects evidenced in Fig. 1 do not depend on the physical mechanisms that bind the electromagnetic energy to the waveguide. In the first section, we examined surface modes that solely result from the coupling between an electromagnetic field and the moving charges of the conducting stripe. However, other families of solutions can also be obtained by either coating the waveguide with a dielectric layer or by structuring the metal surface at the sub-wavelength scale. Both approaches amount at lowering the effective conductivity of the metal surface and therefore they are even valid in the limit of perfectly conducting waveguides. As an example, we consider here a perfect metal stripe on a thin dielectric layer $\left(\mathrm{n}_{\text {diel }}=1.46\right)$ surrounded by air (Fig. $\left.2 \mathrm{a}\right)$. Although the geometry is reminiscent of the structure studied in section 1, the field cannot penetrate in (and thus be bound to) the metal, which changes the nature of the modes in a fundamental way. Here, any bound solution solely results from the presence of the dielectric layer 5 .

We first examine a perfect conductor stripe with a width $w=$ $2 \mu \mathrm{m}$ and a thickness $t=20 \mathrm{~nm}$, i.e. with the same subwavelength dimensions investigated in section 1 . The thickness of the dielectric layer is set to $10 \mu \mathrm{m}(\lambda / 30$ at $1 \mathrm{THz})$. Figs. $2 \mathrm{~b}$ and $2 \mathrm{c}$ show the power distribution, the $\mathrm{E}_{\mathrm{y}}$ field pattern, and a plot of the vectors representing the electric field of the only mode found by our solver at $1 \mathrm{THz}$. One can see that this solution is reminiscent of the $\mathrm{sa}_{\mathrm{b}}{ }^{0}$ mode of the Au stripe studied in section 1 . First, the mode energy is localized near the four corners of the structure, revealing lightning rod effects. Second, the field component $\mathrm{E}_{\mathrm{y}}$ has the same field parity as the $\mathrm{sa}_{\mathrm{b}}{ }^{0}$ mode and crucially it features an axial field distribution. The similarities between this mode and the $\mathrm{sa}_{\mathrm{b}}{ }^{0}$ mode are all the more striking that the waveguides supporting these solutions operate on very different principles. Here the mode has all the properties of subwavelength Goubau waves; i.e. it is bound to a conductor coated with a thin dielectric layer and characterized by an axial field distribution ${ }^{5}$. The importance of the dielectric layer for binding the mode can be seen by the evanescent tail of the energy in the air region beneath the dielectric layer.

The evolution of this bound mode as a function of the metal thickness $t$ for different widths is shown on Fig. $2 \mathrm{~d}$ at $1 \mathrm{THz}$. In all cases, the effective index $\mathrm{n}_{\text {eff }}$ significantly increases as $t$ decreases and the slope of the dispersion curves increases as the waveguide width is smaller, just as in the case of the $\mathrm{sa}_{\mathrm{b}}{ }^{0}$ mode investigated in section 1 . Note however that $\mathrm{n}_{\mathrm{eff}}$ does not diverge as the waveguide transverse dimensions tend to zero. Rather, the effective index approaches finite values, which reflect the fraction of the mode energy localized either in the dielectric layer or in the air. These differences are a direct consequence of the fact that the stripe has an infinite conductivity contrary to the previous case.

Fig. 2e shows a cross-section of the power carried by the mode along the $y$ axis above the stripe (in the air) for stripes with two different sizes. The mode confinement strongly increases as the transverse dimensions of the stripe are reduced to highly sub-wavelength dimensions: for the smallest stripe $(w=2 \mu \mathrm{m}, t=20 \mathrm{~nm})$ the lateral mode extension is reduced down to $2.2 \mu \mathrm{m}$, or $\lambda / 136$.

We calculated the dispersion relation of the modes supported by a $10 \mu \mathrm{m}$-width and $200 \mathrm{~nm}$-thick perfect conducting stripe (Fig. 2f). To keep binding effects constant at all operation frequencies, we set the dielectric layer thickness to $\lambda / 2 \mathrm{n}_{\text {diel }}$. The curves bear many similarities with those computed for the Au stripe of section 1 (Fig. 1f). In particular, the structure supports a variety of modes from the optical to the far-infrared range, and all these solutions are successively going into cutoff as the frequency decreases. The only exception to this general trend is the trajectory of the $\mathrm{THz}$ bound mode (red curve). In contrast to the other solutions, the effective index of this mode increases with decreasing frequencies, and as the frequency reaches the $\mathrm{THz}$ regime the slope of the curves becomes steeper. 


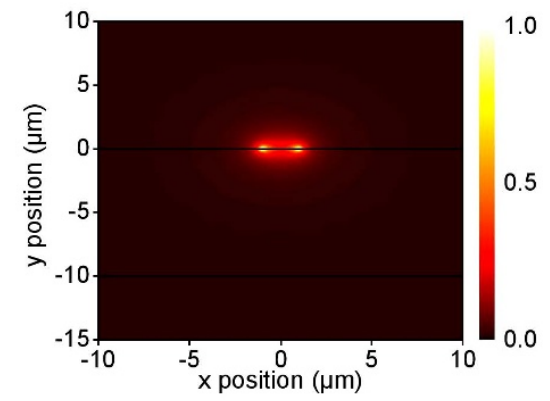

C
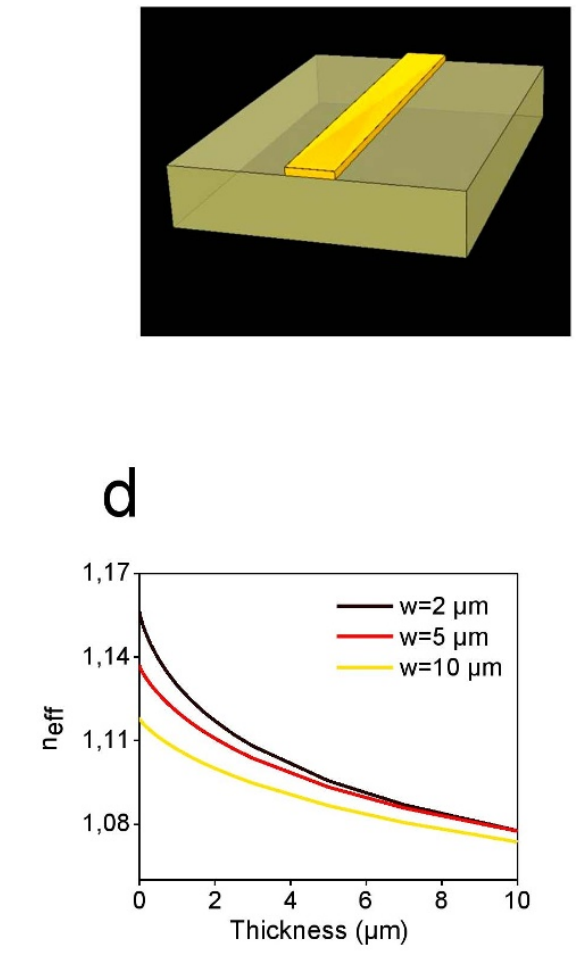
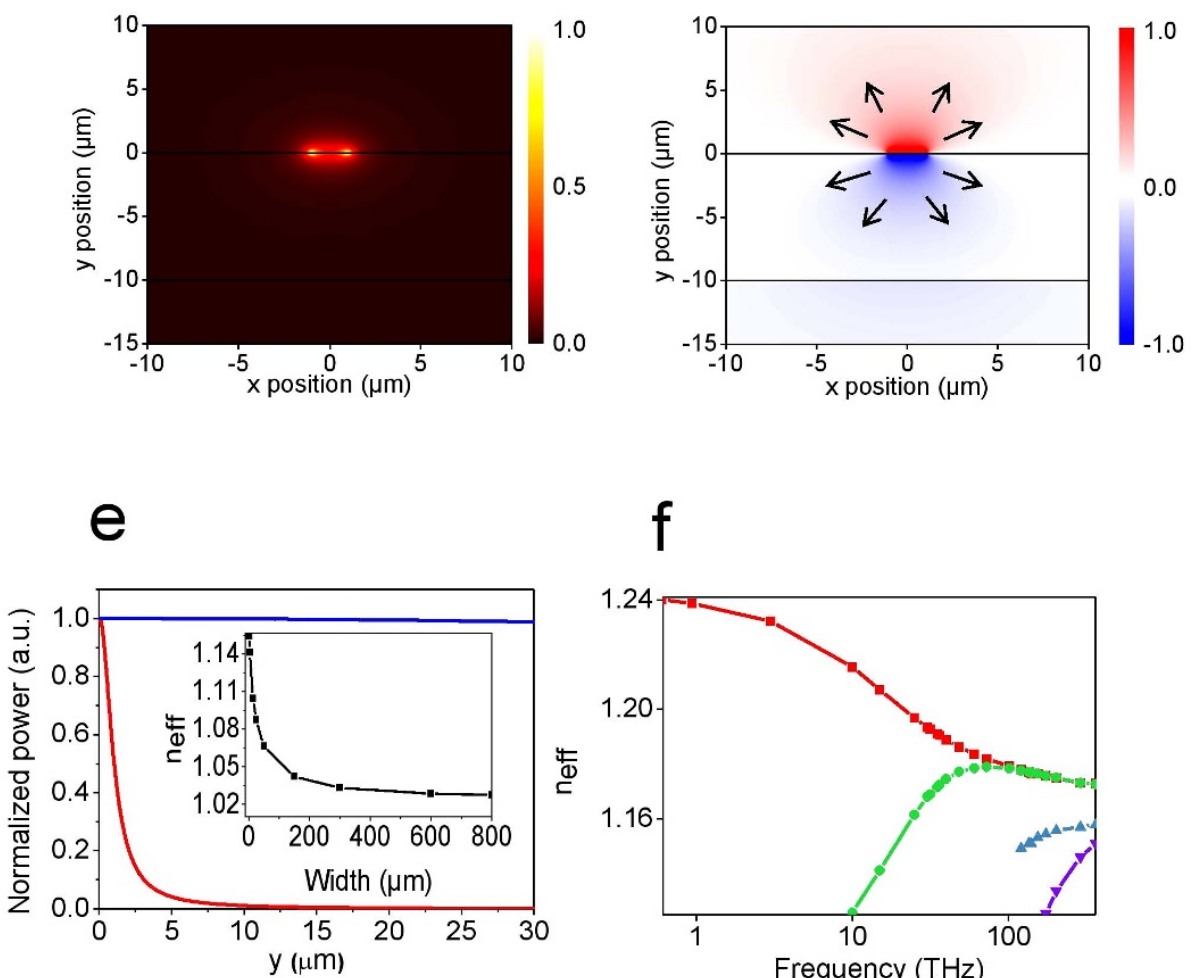

1.0

0
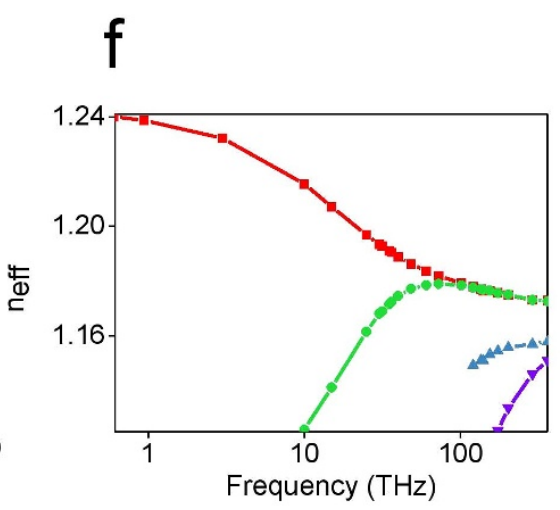

Figure $2 \mid$ Simulation of the modes supported by a perfectly conducting stripe on a thin dielectric layer. (a) Schematic of a rectangular perfect metal stripe on a thin dielectric layer $\left(\mathrm{n}_{\text {diel }}=1.46\right)$ surrounded by air. The dielectric layer is infinitely wide and its thickness is set in the subwavelength range $\left(<\lambda / 2 \mathrm{n}_{\text {diel }}\right)$. (b) Cross-section map representing the power carried by the mode propagating along a perfect conductor stripe with a width $\mathrm{w}=2 \mu \mathrm{m}$ and a thickness $\mathrm{t}=20 \mathrm{~nm}$ at $1 \mathrm{THz}$. The thickness of the dielectric layer is set to $10 \mu \mathrm{m}(\lambda / 30 \mathrm{at} 1 \mathrm{THz})$ since the bound mode only exists in the limit of a dielectric layer thickness that does not exceed the critical value of $\lambda / 2 \mathrm{n}_{\text {diel }}$. No other mode has been found by sweeping the frequency from $0.5 \mathrm{THz}$ to $5 \mathrm{THz}$. (c) Ey field distribution for the same mode. The arrows represent the total field E. (d) Effective index $\mathrm{n}_{\text {eff }}$ as a function of the metal thickness $t$ for different widths of the perfect conducting stripes. (e) Cross-section of the power carried by the mode, in the air, for a large stripe (width $\mathrm{w}=30 \mu \mathrm{m}$, thickness $\mathrm{t}=10 \mu \mathrm{m}$, blue curve) and for a small stripe ( $\mathrm{w}=2 \mu \mathrm{m}, \mathrm{t}=20 \mathrm{~nm}$, red curve). The power has been evaluated along the vertical symmetry plane of the structure (i.e. the y axis) in the upper plan. (f) Dispersion relation of the modes supported by a $10 \mu \mathrm{m}$-width and $200 \mathrm{~nm}$-thick perfect conducting stripe. The red curve shows the evolution of the mode examined in figs. 2a-e; it is the only solution that is not in cutoff in the THz range. To keep binding effects constant at all operation frequencies, we set the dielectric layer thickness to $\lambda / 2 \mathrm{n}_{\text {diel }}$.

Thus, the mechanisms that allowed the $\mathrm{sa}_{\mathrm{b}}{ }^{0}$ mode of the Au stripe to survive at low frequencies (cf. Fig. 1f) are also at work here: the $\mathrm{THz}$ bound mode studied in this section does not have any cutoff because of the reinforcement of the electromagnetic field interactions across the waveguide cross-section and the lightning rod effects. Since the dimensions of the structure become smaller with respect to the wavelength as the frequency decreases, this behavior is another evidence that shrinking the transverse size of metallic waveguides is a powerful strategy to achieve high electric field confinement at $\mathrm{THz}$ frequencies. Note that the size effects evidenced in this figure are not counterbalanced by the dispersive screening effects contrary to the dispersion relations that we obtained for a Au stripe embedded in a homogeneous medium.

Modes supported by a planar Goubau line. We now provide an experimental validation of the key role played by the transverse size of the waveguides by studying a real planar Goubau line made of a finite conductivity metal stripe on a thin dielectric layer (Kapton ${ }^{31,32}$ ). This structure supports hybrid modes that can be seen as a combination of the $\mathrm{as}_{\mathrm{b}}{ }^{0}$ mode studied in section 1 and the $\mathrm{THz}$ bound mode studied in section 2 . The dispersion relation of the modes propagating on planar Goubau lines is measured using a guided wave $\mathrm{THz}$ time domain spectroscopy experiment (see Supplementary
Methods). Figure $3 \mathrm{~b}$ shows the time-domain electric field waveforms measured at the entrance of the line, after $500 \mu \mathrm{m}$ and $1 \mathrm{~mm}$ of propagation, for two different widths $(200 \mu \mathrm{m}$ and $30 \mu \mathrm{m}$, see Supplementary Methods). After $1 \mathrm{~mm}$ of propagation, the peak of the detected $\mathrm{THz}$ waveform propagating on the large line shows a time delay of 538 fs compared to the waveform propagating on the narrow line. This tendency indicates that the phase velocity and consequently the real part of the effective index of the Goubau mode rise as the stripe width becomes smaller. Besides, the waveform is distorted during the propagation, as can be seen with the appearance of a negative peak after the main positive peak, explained by Gibbs phenomenon in the time domain ${ }^{27}$.

From the Fourier transforms of the measured time-domain waveforms, the effective index of the Goubau modes can be extracted. Figure $3 c$ shows the real part of the effective index of the modes as a function of the frequency for the two different widths and compares these data with numerical simulations. Note that the simulations assume a dispersionless dielectric layer. The good agreement between the numerical and experimental data points validates this approximation and indicates that the dispersive effects are dominated by the metal conductivity and the size of the metal stripe and of the dielectric slab. These results demonstrate that the reduction of the stripe width from $200 \mu \mathrm{m}$ to $30 \mu \mathrm{m}$ significantly increases 
a

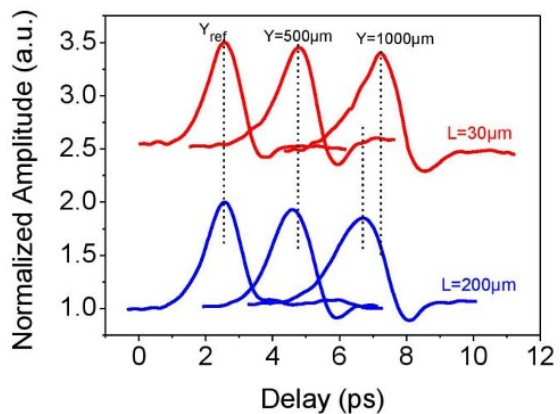

b

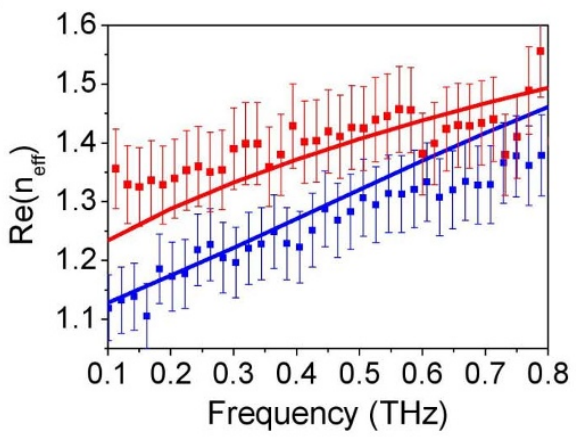

Figure $3 \mid$ Measurements of the mode properties supported by a planar Goubau line. (a) Time-domain electric field waveforms at the entrance of the planar Goubau line, after propagating $500 \mu \mathrm{m}$ and $1 \mathrm{~mm}$ on planar Goubau lines with widths of $200 \mu \mathrm{m}$ and $30 \mu \mathrm{m}$. (b) Experimental (squares) and simulated (lines) real part of the effective index of the modes propagating on planar Goubau lines of width $200 \mu \mathrm{m}$ (blue) and $30 \mu \mathrm{m}$ (red) as a function of the frequency. The error bars denote uncertainties in experimental measurement.

the real part of the effective index and thus improves the localization of the electromagnetic energy around the metal surface. We can also derive the group dispersion velocity from the measured waveforms, a critical characteristic of waveguides for $\mathrm{THz}$ pulse propagation. At $0.6 \mathrm{THz}$, the group velocity dispersion is $0.4 \mathrm{ps}^{2} / \mathrm{mm}$ and $0.23 \mathrm{ps}^{2} / \mathrm{mm}$ for stripe width of $200 \mu \mathrm{m}$ and $30 \mu \mathrm{m}$ respectively, indicating a slight decrease of the group velocity as the width of the stripe is reduced. The imaginary part of the effective index is hardly accessible using our experimental set up sensitivity since its does not exceed 0.013 as confirmed by the simulations.

\section{Discussion}

$\mathrm{THz}$ surface waves can exhibit extreme sub-wavelength confinement (smaller than $\lambda / 100$ ) by simply shrinking the transverse dimensions of the structure along which they propagate. This size effect must be distinguished from the physics defining the nature of the mode (i.e. Sommerfeld wave, Goubau wave, Spoof Plasmons...). For large waveguides, the mode properties are primarily governed by the mechanisms that bind the mode to the metal surface: the dispersion, electromagnetic confinement and field symmetries strongly depends on the metal conductivity, the presence of sharp corners, and any factor that may change the effective parameters of the surface (dielectric coating, metallic corrugations, surface roughness...). However, for small waveguides, the size effects cannot be neglected anymore and as the waveguide cross-section tends to the nanoscale, size effects can be used as a simple yet formidable tool for high electric field confinement at $\mathrm{THz}$ frequencies. It would be interesting to generalize this result into a unified theory. This is beyond the scope of this paper and it will be the subject of a future publication. Understanding this universal behavior should significantly ease the design of highly miniaturized $\mathrm{THz}$ components, and particularly when it comes to optimizing the trade-off between field confinement and absorption losses for a given application. More importantly, the fact that simple geometries support surface modes with a field confinement comparable to the wavelength of light blurs the boundaries between $\mathrm{THz}$ science and nano-optics, opening up exciting new avenues for near-field imaging, single molecule detection, and ultrafast nonlinear phenomena.

\section{Methods}

Simulation. We assume that the metal waveguide is infinitely long along the propagation direction $z$ so we expect solutions of the form $E=E_{0}(x, y) \exp \left(i . n_{\text {eff }} k_{0} z\right)$, where $\mathrm{E}_{0}$ is the electric field in the transverse $x y$ plane, $\mathrm{n}_{\mathrm{eff}}$ is the complex effective index and $\mathrm{n}_{\text {eff }} \mathrm{k}_{0}=\mathrm{n}_{\text {eff }} 2 \pi v$ is the wavevector of the mode. Although this problem can be formulated in two dimensions, it is well known that the rectangular geometry does not admit analytical solutions. In this article we solve for the modes of the structure by performing an eigenmode analysis with a finite element package (Comsol multiphysics). Due to the very high permittivity of $\mathrm{Au}$ in the $\mathrm{THz}$ range (bottom inset of Fig. 1f), a special care must be taken to mesh the computational domain properly. In particular, the discretization must be smaller than the skin depth of Au in the vicinity of the metal interface, requiring elements whose size is significantly smaller than $100 \mathrm{~nm}$, or $\sim \lambda / 1000$, around the metal waveguide. The conducting properties of $\mathrm{Au}$ are modeled by using a complex permittivity fitting the experimental values compiled in Ref. 33, represented in the bottom inset of Fig. 1f. In the simulations involving perfect conductors, we only included the walls of the stripe in the model since the field cannot penetrate inside. In this case, there is no dissipation and the effective index $n_{\text {eff }}$ is real.

Measurements. The guided-wave time domain spectroscopy (THz-TDS) experiment makes use of a coherent detection of the electric field, enabling the direct determination of the dispersion relation of the propagating modes. In the experimental setup, which is similar to that described in our earlier publication ${ }^{27}$, the $\mathrm{THz}$ pulses are directly generated and detected on waveguides thus avoiding the usual problem of the coupling efficiency existing in free space THz-TDS. The propagation characteristics of the $\mathrm{THz}$ pulses are measured by moving an electro-optic detector along the axis of the line and just above its center.

Devices. The planar Goubau lines are fabricated on a $58 \mu \mathrm{m}$-thick dielectric layer of Kapton with refractive index of $1.85^{31,32}$. The rectangular cross-section of the single conductor is made of Ti/Au $(20 / 500 \mathrm{~nm})$ and is 4-mm-long. Two distinct line widths of $30 \mu \mathrm{m}$ and $200 \mu \mathrm{m}$ are defined, all other parameters being identical. The excitation of the Goubau mode is ensured by a coplanar waveguide (central stripe width of $12.5 \mu \mathrm{m}$, ground planes widths of $400 \mu \mathrm{m}$, and slots size of $2 \mu \mathrm{m}$ ) connected to the planar Goubau line using tapered sections that provide smooth impedance transformation between the coplanar waveguide and the planar Goubau line.

1. Sommerfeld, A. Über die Ausbreitung der Wellen in der drahtlosen Telegraphie. Ann. Phys. 333, 665-736 (1909).

2. Jeon, T. I., Zhang, J. \& Grischkowsky, D. THz Sommerfeld wave propagation on a single metal wire. Appl. Phys. Lett. 86, 161904 (2005).

3. Zenneck, J. Über die Fortpflanzung ebener elektromagnetischer Wellen längs einer ebenen Leiterfläche und ihre Beziehung zur drahtlosen Telegraphie. Ann. Phys. 328, 846-866 (1907).

4. Barlow, H. M. Surface Waves. Proc. IRE 46, 1413-1417 (1958).

5. Goubau, G. Surface waves and their application to transmission lines. J. Appl. Phys. 21, 1119-1128 (1950).

6. Harvey, A. F. Periodic and guiding structures at microwave frequencies. IEEE Trans. Microwave Theory Techn. 8, 30-61 (1960).

7. Ulrich, R. \& Tacke, M. Submillimeterwaveguiding on periodic metal structure. Appl. Phys. Lett. 22, 251-253 (1973)

8. Pendry, J. B., Martín-Moreno, L. \& García-Vidal, F. J. Mimicking surface plasmons with structured surfaces. Science 305, 847-848 (2004).

9. Alastair, P. H., Benjamin, R. E. \& Sambles, J. R. Experimental verification of designer surface plasmons. Science 308, 670-672 (2005).

10. Williams et al. Highly confined guiding of terahertz surface plasmonpolaritons on structured metal surfaces. Nature Photon. 2, 175-179 (2008).

11. Maier, S. A., Andrews, S. R., Martin-Moreno, L. \& Garcia-Vidal, F. J. Terahertz surface plasmon-polariton propagation and focusing on periodically corrugated metal wires. Phys. Rev. Lett. 97, 176805 (2006).

12. Schlesinger, Z. \& Sievers, A. J. IR surface-plasmon attenuation coefficients for Gecoated Ag and Au metals. Phys. Rev. B 26, 6444-6454 (1982).

13. Steijn, K. W., Seymour, R. J. \& Stegeman, G. I. Attenuation of far-infrared surface plasmons on overcoated metal. Appl. Phys. Lett. 49, 1151-1153 (1986).

14. Gong, M., Jeon, T.-I. \& Grischkowsky, D. THz surface wave collapse on coated metal surfaces. Opt. Express 17, 17088-17101 (2009). 
15. Saxler, J. et al. Time-domain measurements of surface plasmonpolaritons in the terahertz frequency range. Phys. Rev. B 69, 155427 (2004).

16. Huber, A. J., Keilmann, F., Wittborn, J., Aizpurua, J. \& Hillenbrand, R. Terahertz near-field nanoscopy of mobile carriers in single semiconductor nanodevices. Nano Lett. 8, 3766-3770 (2008).

17. Deibel, J. A., Escarra, M., Berndsen, N., Wang, K. \& Mittleman, D. M. Finiteelement method simulations of guided wave phenomena at terahertz frequencies. Proc. IEEE 95, 1624-1640 (2007)

18. Seo, M. A. et al. Terahertz field enhancement by a metallic nano slit operating beyond the skin-depth limit. Nature Photon. 3, 152-156 (2009).

19. Shalaby, M. et al. Concurrent field enhancement and high transmission of $\mathrm{THz}$ radiation in nanoslit arrays. Appl. Phys. Lett. 99, 041110 (2011).

20. Astley, V., Mendis, R. \& Mittleman, D. M. Characterization of terahertz field confinement at the end of a tapered metal wire waveguide. App. Phys. Lett. 95, 031104 (2009).

21. Yang, J., Cao, Q. \& Zhou, C. Theory for terahertz plasmons of metallic nanowires with sub-skin-depth diameters. Opt. Express 18, 18550-18557 (2010).

22. Liu, J., Mendis, R., Mittleman, D. M. \& Sakoda, N. A tapered parallel-platewaveguide probe for $\mathrm{THz}$ near-field reflection imaging. Appl. Phys. Lett. 100 031101 (2012).

23. Zhan, H., Mendis, R. \& Mittleman, D. M. Superfocusing terahertz waves below $\lambda / 250$ using plasmonic parallel-plate waveguides. Opt. Express $18,9643-9650$ (2010).

24. Zhan, H., Mendis, R. \& Mittleman, D. M. Characterization of the terahertz nearfield output of parallel-plate waveguides. J. Opt. Soc. Am. B 28, 558-566 (2011).

25. Dazhang, L. et al. On-chip terahertz Goubau-line waveguides with integrated photoconductiveemitters and mode-discriminating detectors. Appl. Phys. Lett. 95, 092903 (2009)

26. Treizebre, A. \& Bocquet, B. Nanometric metal wire as a guide for $\mathrm{THz}$ investigation of living cells. Int. J. Nanotechnol. 5, 784-795 (2008).

27. Gacemi, D. et al. THz surface plasmon modes on planarGoubaulines. Opt. Express 20, 8466-8471 (2012).

28. Berini, P. Plasmon-polariton waves guided by thin lossy metal films of finite width: Bound modes of symmetric structures. Phys. Rev. B 61 10484-10503 (2000).

29. Berini, P. \& De Leon, I. Surface plasmon-polariton amplifiers and lasers. Nature Photon. 6, 16-24 (2011).

30. Wang, K. \& Mittleman, D. M. Dispersion of surface plasmonpolaritons on metal wires in the THz frequency range. Phys. Rev. Lett. 96, 157401 (2006).
31. Walther, M. et al. Terahertz metamaterials fabricated by inkjet printing. Appl. Phys. Lett. 95, 251107 (2009).

32. Cunningham, P. D. et al. Broadband terahertz characterization of the refractive index and absorption of some important polymeric and organic electro-optic materials. J. Appl. Phys. 109, 043505 (2011).

33. Ordal, M. A. et al. Optical properties of the metals $\mathrm{Al}, \mathrm{Co}, \mathrm{Cu}, \mathrm{Au}, \mathrm{Fe}, \mathrm{Pb}, \mathrm{Ni}, \mathrm{Pd}$, $\mathrm{Pt}, \mathrm{Ag}, \mathrm{Ti}$, and $\mathrm{W}$ in the infrared and far infrared. Appl. Opt. 22, 1099-1120 (1983).

34. Berini, P., Charbonneau, R., Lahoud, N. \& Mattiussi, G. Characterization of longrange surface-plasmon-polariton waveguides. J. Appl. Phys. 98, 043109 (2005).

35. Lynch, D. W. \& Hunter, W. R. Comments on the optical constants of metals and an introduction to the data for several metals. Handbook of Optical Constants of Solids volume 1 (Palik, E. D. Ed, Academic Press: New York 1985).

36. Johnson, P. B. \& Christy, R. W. Optical constants of the noble metals. Phys. Rev. B 6, 4370-4379 (1972).

\section{Acknowledgements}

We thank P. Crozat for fruitful discussion. This work was supported by EMRP Researcher Grant NEW07 REG3 related to the Joint Research Project 'THz Security'.

\section{Author contributions}

D.G. and A.D. performed the numerical simulations, D.G. and J.M. performed the experiments, D.G., J.M., R.C. and A.D. analyzed the data, J.M. and A.D. wrote the manuscript. J.M., R.C. and A.D. developed the narrative and major concepts based on an original idea by J.M.

\section{Additional information}

Competing financial interests: The authors declare no competing financial interests. License: This work is licensed under a Creative Commons Attribution-NonCommercial-NoDerivs 3.0 Unported License. To view a copy of this license, visit http://creativecommons.org/licenses/by-nc-nd/3.0/

How to cite this article: Gacemi, D., Mangeney, J., Colombelli, R. \& Degiron, A. Subwavelength metallic waveguides as a tool for extreme confinement of $\mathrm{THz}$ surface waves. Sci. Rep. 3, 1369; DOI:10.1038/srep01369 (2013). 
SUBJECT AREAS: $\quad$ ERRATUM: Subwavelength metallic waveguides as a tool for extreme TERAHERTZ OPTICS confinement of $\mathrm{THz}$ surface waves

ULTRAFAST PHOTONICS NANOPHOTONICS AND PLASMONICS SUB-WAVELENGTH OPTICS D. Gacemi, J. Mangeney, R. Colombelli \& A. Degiron

Due to a technical error, the original Article contained errors in the numbering of several papers in the reference list and two references were omitted. This has now been corrected in the Article.

\section{SCIENTIFIC REPORTS:}

$3: 1369$

DOI: $10.1038 /$ srep0 1369

Published: 6 March 2013

Updated: 28 March 2013 